\title{
Chimeric antigen receptor-T cell, a novel player in anti-tumor treatment
}

\author{
Jieming $\mathrm{Ni}^{1}$, Anping $\mathbf{N i}^{2}$ \\ ${ }^{1}$ Seven year program of China Medical University, Shenyang 110122, China; ${ }^{2}$ Department of Clinical Laboratories, Peking Union Medical College \\ Hospital, Chinese Academy of Medical Sciences \& Peking Union Medical College, Beijing 100730, China \\ Contributions: (I) Conception and design: A Ni; (II) Administrative support: None; (III) Provision of study materials or patients: None; (IV) \\ Collection of data and data analysis: A Ni, J Ni; (V) Data analysis and interpretation: None; (VI) Manuscript writing: All authors; (VII) Final approval \\ of manuscript: All authors. \\ Correspondence to: Prof. Anping Ni. Department of Clinical Laboratories, Peking Union Medical College Hospital, Chinese Academy of Medical \\ Sciences \& Peking Union Medical College, Beijing 100730, China. Email: cotton1995@163.com.
}

\begin{abstract}
Chimeric antigen receptor (CAR)-T cells reprogram the $\mathrm{T}$ cells to target tumor cells and have undergone several generations of enhancement. Accumulating evidence shows that by incorporating co-stimulatory molecules and single-chain variable fragments (scFvs), CAR-T cells maintain sustainable proliferation capacity along with high sensitivity and enhanced cytokines production. Moreover, CAR-T cell therapy has shown great promise in clinical trials. However, complications and toxicities must be considered and managed. In this review, we aimed to summarize the development of CAR and the criteria for the selection of the tumor associated antigen (TAA) based on the literature. In addition, we review current clinical trials of CAR-T therapy. Finally, we discuss the adverse effects of CAR-T therapy, managements of complications, and expectations from CAR-T therapy.
\end{abstract}

Keywords: Antigen; immunotherapy; receptors; T-cell

Submitted Feb 27, 2018. Accepted for publication Jun 01, 2018.

doi: $10.21037 /$ tcr.2018.06.11

View this article at: http://dx.doi.org/10.21037/tcr.2018.06.11

\section{Introduction}

$\mathrm{T}$ cell plays a significant role in anti-tumor immunity, and TCR executes immune function by cooperating with CD4/8 and co-stimulatory signals. CAR-T cells are genetically engineered $\mathrm{T}$ cells with the expression of tumor-targeting receptors and increased cytotoxic potency to target cells, which have undergone several generations of enhancement. CAR-T cells recognize tumor antigens without MHC restriction and the aid of antigen presenting cells (APCs). CAR is composed of the extracellular, hinge, transmembrane and intracellular domains. The intracellular domain is mostly $\mathrm{CD} 3 \zeta$ or $\mathrm{FcR} \gamma$, however, there are more investigations regarding the $\mathrm{CAR}$ attached with $\mathrm{CD} 3 \zeta$, due to its quantitative superiority of intracellular ITAMs. The transmembrane domain mostly consists of $\mathrm{CD} 3 \varepsilon, \mathrm{CD} 8$ or $\mathrm{CD} 28$, which also influences the expression and anti-tumor capability of CAR. Currently, CD19, Her-2, ROR1, CD20, etc. are common scFv targets in the extracellular domain. CAR-T cell immunotherapy has made unprecedented progress in acute lymphoblastic leukemia (ALL) treatment, among which B-ALL is the most prevalent type in children. The relapse-free survival rate of adolescents and children with ALL, who received CAR-T therapy, reached more than $50 \%$. And complete response rate reached over $90 \%$ with long persistence. However, prognosis in the elderly and children with B-ALL was poor, with about 10-20\% suffering from relapse, demonstrating that CAR-T therapy still requires refinement (1-3). Adverse effects such as cytokines release syndrome and tumor lysis syndrome are also obstacles to CAR-T application. Therefore, it is imperative to identify and design appropriate management strategies for all complications and toxicities associated with CAR-T therapy. In this review, we propose several measures in order to improve CAR-T cell construction so as to minimize the potential risks related to CAR-T therapy. 
Table 1 Criteria for selecting tumor associated antigens

Characteristics
Antigens excessively expressed in tumor and remaining low
level in normal tissues (6-9)
Proteins showed abnormalities in structure or expression
(10,11)
Antigens mainly expressed in tumor cells (12,13)
Viruses (14,15)
Mesenchymal molecules (16)
Sexual differentiation related antigens (17)
Example
gp100, MART, Her-2
p53, GM2, Ras, MAGE, MUC-1
BRAF, EGFR VIII
EBV, HBV
VEGF Receptor
NY-ESO-1
EBV, Epstein-Barr virus; EGFR, epidermal growth factor receptor;
HBV, Hepatitis B virus; Her-2, human epidermal growth factor
receptor-2; MAGE, melanoma antigen gene; MART, melanoma
antigen recognized by T cells; MUC-1, Mucin 1; VEGF, vascular
endothelial growth factor.

In future, a more refined CAR-T therapy would offer substantial advancements in anti-tumor practice.

\section{CAR-T cell preparation and design}

CAR-T cell construction is a comprehensive discipline, which incorporates immunology and gene engineering. Transfection via electroporation or viral vectors is used to introduce CAR into T or NK cells. Generally, T cells are isolated from peripheral blood, and most of CAR-T treatments initially involve leukapheresis, which is followed by IL-2 administration or CD3/CD28 microbead expansion ex vivo (4). Leukapheresis reduces GVHD risk, achieves cell-mediated cancer regression, and increases anti-tumor efficacy. The amplified $\mathrm{T}$ cells are finally re-infused into the autologous host after radiation therapy and chemotherapy, both of which lead to great toxicity. Recent clinical trials preferred retroviral and lentiviral vectors as transgenes for CAR-T construction. However, screening for potent viral vectors for replication will inevitably bring about high cost. Compared with viral vectors, the introduction of transposons into cells by electroporation provides a stable, easy and inexpensive method for CAR-T cell construction (5).

Another issue concerning CAR-T cell preparation is the selection of an appropriate TAA. Several common criteria are summarized below based on antigen characteristics. (I) Compared with the low expression level in normal tissue, several differentiation-related antigens, such as Her-2, gp100, and MART, are overexpressed in breast carcinoma and melanoma, which can serve as therapeutic targets $(6,7)$. Among them, Her-2 has recently become a hotspot in CAR-T research. Her-2 inhibitor, trastuzumab, offers a novel therapeutic approach against the EGFR mediated pathway in lung cancer (8). Four of the 17 patients who received Her2-CAR infusion $\left(1 \times 10^{4} / \mathrm{m}^{2}\right.$ to $1 \times 10^{8} / \mathrm{m}^{2}$ ) had stable diseases for a period of 12 weeks14 months, and the median overall survival time was 10.3 months (NCT00902044) (9). (II) Proteins that showed abnormalities in structure or expression, including MUC-1, Ras and p53, may be tightly related to CAR expression and regarded as specific antigens $(10,11)$. (III) Antigens mainly presented in tumor cells, including BRAF in melanoma and EGFR VIII mutation in breast carcinoma, variably affect CAR expression and could be selected to minimize impairment $(12,13)$. (IV) Viruses like HBV can be rationally treated as CAR targets. EBV specific CTLs with anti-Her2 CAR expression can function as tumor directed effector cells and survive longer in vivo, due to persistent stimulation from viral antigens $(14,15)$. (V) Mesenchymal molecules like VEGF receptor, would become candidate targets (16), however, it may be associated with irreversible hematopoietic injury. (VI) Cancer-testis antigens like NYESO-1, which are only expressed in embryonic period, are preferred in multiple myeloma treatment because of their low expression levels after puberty (17) (Table 1).

The first generation of CAR was mainly composed of two parts, an extracellular scFv and an intracellular $\mathrm{CD} 3 \zeta$. Due to the lack of co-stimulatory signals, this generation of CAR-T cells could not proliferate continuously and produce cytokines sufficiently, therefore the anti-tumor effect was not very good. The second generation of CAR made great breakthroughs because of the intracellular co-stimulatory signals provided by 4-1BB and OX40, etc. (18). However, contrary to the success in vitro, second generation of CAR-T cells did not stably proliferate to produce sufficient IL-2 in vivo, because of the lack of exogenous signals. And supplementation of exogenous 4-1BB or replacement with third generation facilitated massive proliferation of CAR-T cells (19). The third generation integrates multiple co-stimulatory molecules and produces cytokines intensively. Based on preclinical results, the third generation attached with both 4-1BB and CD28 presents superiority to second 


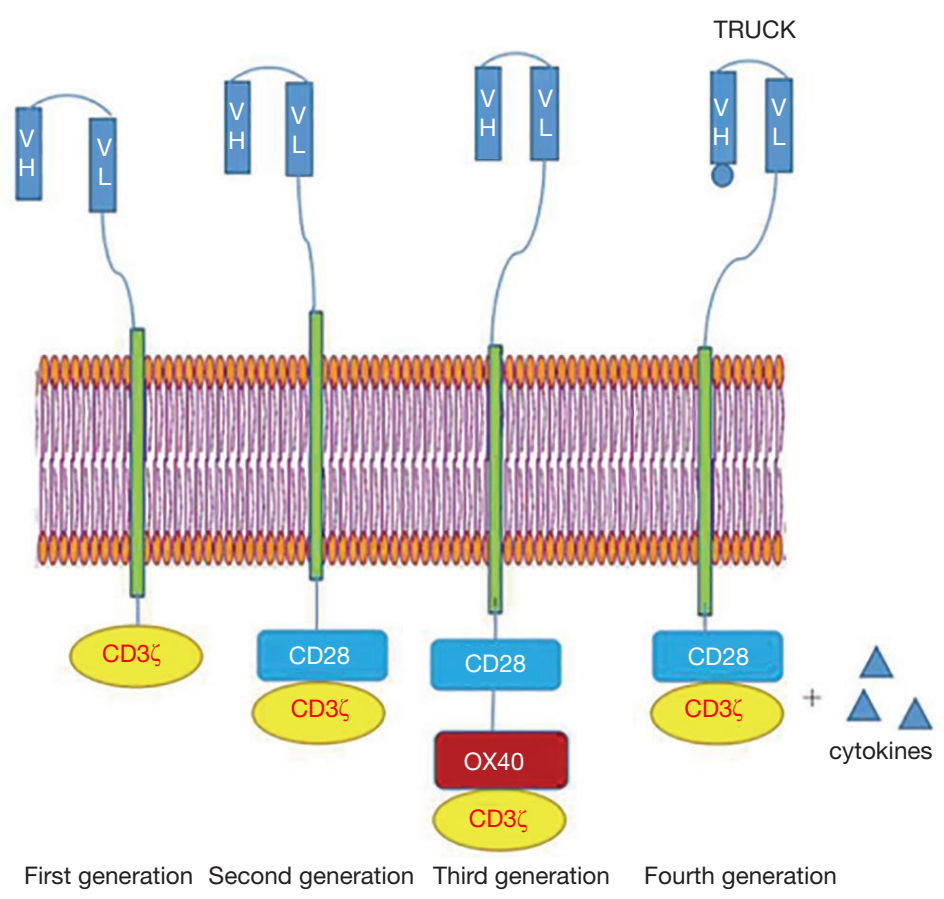

Figure 1 The first generation of CAR is mainly composed of $\mathrm{CD} 3 \zeta$ and scFv. The second generation additionally introduces co-stimulatory signals. The third generation contains multiple co-stimulatory molecules. The fourth generation is updated with promoters and releases cytokines intensively. $\mathrm{VH}$, variable region of heavy chain; VL, variable region of light chain.

generation attached only with CD28 (20). However, apart from co-stimulatory molecules, hinge, intracellular and transmembrane domains will also influence the clinical effects of CAR-T cells, so it remains unknown whether the third generation is more capable in tumor treatment. Tumor cells exhibit heterogeneity in antigens, therefore antigennegative tumor cells tend to proliferate and enter into relapse in CAR-T therapy. The fourth generation (TRUCK) is specifically designed with promoters or NFAT responsive expression cassette to redirect the tumor microenvironment, deal with disadvantages of pre-conditioning, and prevent antigen negative relapse. TRUCKs actively release cytokines to prolong persistence, recruit more immune cells and enhance therapeutic index (21). Pegram et al. (22) have designed an anti-CD19 CAR with a good anti-tumor effect, which releases cytokines, augments T-cell activation, and stimulates innate immune cells to mitigate immune escape (21). Figure 1 In addition, Tandem CAR-T cell will provide a fresh outlook for tumor treatment, which may be attributed to high selectivity, optimized cytokine production, and limited tumor escape. Tandem CAR exodomain (TanCAR) introduces double CARs simultaneously or only comprises a single CAR targeting bi-specific antigen, which increases sensitivity and mitigates immune escape. Hegde et al. (23) have created a CAR that incorporates CD28 $\zeta$ as an endodomain with the Her-2/IL13R $\alpha 2$ compound as an exodomain. And a patient who received Tandem CAR-T cells had capability to lyse glioblastoma. TanCAR binding with CD19/CD20 demonstrated that single vector targeting bi-specific antigens was more effective to control tumor and less toxic in the high disease burden group (24). This indicates the possibility that TanCAR can play a pivotal role in overcoming challenges associated with anti-tumor therapy.

\section{CAR-T cell immunotherapy in clinical trial}

\section{Clinical efficacy of CAR-T therapy}

Currently, CAR-T therapy has achieved success in hematogenous tumor treatment. (I) CD19-specific CAR-T immunotherapy induced complete remissions of $\mathrm{B}$ cell lymphoma with long durability and decreased relapse. From 2009 to 2015, 6 patients with ALL and 14 patients with chronic lymphocytic leukemia (CLL) received anti-CD19 CAR-T therapy. The overall response rate of the CLL subjects reached $42.9 \%$, and the complete remission rate of the ALL subjects reached 83.4\% (NCT01029366). Among 
Table 2 Tumor associated antigens applied in clinical trial

\begin{tabular}{ll}
\hline TAA & Targeted tumor \\
\hline Her-2 (6) & Breast carcinoma \\
MUC-1 (11) & Pancreatic and prostate cancer \\
CD19 (25,26) & B cell lymphoma, acute lymphoblastic leukemia \\
CD33 (27) & Acute myeloid leukemia \\
CD30 (28) & Hodgkin lymphoma \\
PSMA (29) & Prostate carcinoma \\
CEA (30) & Rectal and colon carcinoma \\
GD2 (31) & Neuroblastoma, melanoma \\
\hline
\end{tabular}

CEA, carcinoembryonic antigen; Her-2, human epidermal growth factor receptor-2; MUC-1, Mucin 1; PSMA, prostate specific membrane antigen; TAA, tumor associated antigen.

patients with non-Hodgkin lymphoma (NHL), $25 \%$ of the participants who received CD19 specific CAR-T cells had partial responses, and $38 \%$ were identified with complete responses (25). Furthermore, infusion of anti-CD19 CAR-T cells in combination with SCT and vaccine could safely enhance persistence in relapsed pediatric ALL (26). (II) CD33-targeted CAR-T cells effectively ameliorated acute myeloid leukemia (AML), and complete remissions were discovered among $30 \%$ of patients with relapsed/refractory leukemia in phase 1 (NCT01902329) (27). (III) Moreover, after administration of anti-CD30 CAR-T therapy in 7 patients with relapsed Hodgkin lymphoma, complete response was observed in 1 patient, and 3 patients showed transient stable conditions (NCT01316146) (28). (IV) VEGFR is a vital molecule involved in tumor growth, and anti-VEGFR1 CAR-T cell exhibits both cytolytic effect and anti-angiogenic potency (16). From 2010 to 2016, patients with melanoma and renal cancer received anti-VEGFR CAR-T therapy, plus with cyclophosphamide, aldesleukin, and fludarabine. Consequently, 1 out of 4 patients in a cohort (infusion within $1 \times 10^{10}$ cells) demonstrated a partial response (NCT01218867). (V) Presently, CAR-T clinical trials are being conducted for the treatment of solid tumors, mainly PSMA for prostate carcinoma, CEA for colon carcinoma, and GD2 for melanoma (29-31). Two patients in a 5-individual cohort had partial responses with a decline in PSA levels (NCT01929239) (29). Furthermore, 7 patients who received anti-CEA CAR-T cells remained stable conditions, and one was alive 56 months post infusion (NCT01212887) (30). Currently, iC9-GD2 CAR-T cell is being tested among patients with neuroblastoma (NCT01822652) (Table 2).

\section{Complications and related managements}

(I) CAR antigens are possibly expressed or have a common segment in normal tissues, this inevitably leads to off target effect, thereby causing multiple systemic organ failure. A recent research has revealed that after administration of Her-2 specific CAR-T therapy, a patient with colon cancer later manifested with pulmonary infiltrates and respiratory failure (32). Furthermore, $\mathrm{F}_{\mathrm{c}} \mathrm{R}$ is vastly overexpressed in CLL and naturally becomes a perfect therapeutic target. However, F $\mu$ R-CAR therapy leads to elimination of healthy B cells with Fcr segment. (II) Following CAR-T cell transfusion, activated $\mathrm{T}$ cells release inflammatory mediators and large amounts of tumor cells undergo necrosis: this is referred as cytokines release syndrome and tumor lysis syndrome respectively. Patients would manifest with fever, chills, leukocytopenia, anemia, neurotoxicity and hypotension, etc. $(33,34)$ (Table 3). It has been revealed that multiple-times infusion of mesothelin specific CAR-T cells resulted in fatal hypersensitivity and cardiac arrest in the patient with mesothelioma (35). The release of large amounts of tissue factors also contributes to high incidence of coagulation disturbance and rapid accumulation of uric acid, which results in kidney failure, hyperkalemia, and other fatal complications (36). (III) Neurotoxicity is one of the major complications of CAR-T immunotherapy. Endothelial cells in the central nervous system maintain the integrity of the blood brain barrier (BBB). However, CAR-T cell activation induces inflammatory mediators, which stimulate pericytes, enhance endothelial permeability, and increase vascular coagulation (34). The disruption of the BBB finally results in cerebral hemorrhage and edema, contributing to high morbidity and mortality among patients. Therefore, the anti-IL-6 agent, siltuximab, which has demonstrated superiority to tocilizumab in penetrating the CNS, is recommended. Besides, plasma exchange and platelet transfusion serve as strategies for controlling coagulation degree and cytokines levels (37).

Concerning managements of complications and toxicities, before CAR-T cell infusion, precautionary measures should be taken. For example, medical doctors may give NSAIDs instead of glucocorticoids to prevent fever and anaphylaxis. Besides, patients with high-tumor-burden lymphoma, who received allopurinol followed by cyclophosphamide, would show tolerance and no evidence of TLS was identified (38). To prevent potential risks in CAR-T immunotherapy, we propose the following measures. (I) To avoid hypersensitivity and CRS, we advocate a low dosage but multiple-times infusion of CAR-T cells to induce tolerance and memory in the central immune system. The dosage of universal cytokines like IL-2 should be reduced to make the therapy 
Table 3 Adverse effects of CAR-T immunotherapy

\begin{tabular}{ll}
\hline Region & Manifestation \\
\hline Overall manifestation (33) & Fever, chills, fatigue \\
Respiratory system $(32,33)$ & Respiratory failure, pulmonary edema, dyspnea \\
Nervous system (33,34) & Convulsion, aphasia, fatigue, headache, seizures, encephalopathy \\
Cardiovascular system (33) & Cardiac arrest, hypotension, decreased cardiac output, cardiac dysfunction \\
Hematologic system (33) & Leukocytopenia, lymphohistiocytosis, anemia, hypofibrinogenemia \\
Digest system (33) & Vomiting, nausea \\
Renal system (33) & Renal impair, renal failure, azotemia \\
\hline
\end{tabular}

more safe and controllable. The IL-6 receptor inhibitor tocilizumab is used as standard practice to reduce cytokines levels in ALL patients. (II) In terms of hypotension, blood volume can be maintained by administrating saline or vasopressor agents. (III) Regarding the hematopoietic system defects induced by cytokine disorder, rituximab or alemtuzumab can be used to temporarily deplete $\mathrm{T}$ cell with no interference in the CAR-T function as a whole (39). (IV) With respect to leukocytopenia, the colony stimulating factor, filgrastim, should be considered for stimulating leukocyte production. (V) Lastly, CD19positive CAR-T cell immunotherapy impairs healthy B cells to cause hypo-gammaglobulinemia and B cell exhaustion. $\gamma$-immunoglobulin and antibiotics can be used to intermittently alleviate the B cell defect and prevent potential infections. After administrating CAR-T cell therapy, patients must stay in hospital for routine examination of blood and urine according to National Cancer Institute. In addition, medical doctors should monitor vital signs, including temperature, pulse, respiration, and blood pressure.

\section{Future perspectives}

The clinical efficacy of CAR-T therapy has become more prominent in hematogenous tumor treatment. However, compared to the accomplishments made with antileukemia agents, CAR-T therapy encounters a deadlock in the treatment of solid tumors, and the high risks of complications make it a dilemma. With regard to these factors, we here discuss some expectations towards future development: (I) In clinical treatment, medical doctors should sketch out an anti-tumor plan both independently and individually. Due to tumor heterogeneity in plasticity, phenotype and drug resistance, CAR-T cell immunotherapy may not be so sensitive and highly effective. Therefore, unique targets of tumor should be selected, real time patient status should be taken into consideration, and clinical complications should be disposed of in time. (II) Combination therapy may open the new chapter for CAR-T therapy. (i) $\mathrm{T}$ cell homing needs to penetrate the mesenchyme, where stromal cells and mesenchymal stem cells (MSC) may produce fibroblast activation protein (FAP), facilitate tumor metastasis, and induce immune suppressive environment (40). Inducible co-stimulators of CAR-T cell, such as MyD88 with CID binding domain, are helpful to maintain $\mathrm{T}$ cell activation, and CAR-T cell with high expression of heparanase can enhance $\mathrm{T}$ cell trafficking (41,42). (ii) Bruton's tyrosine kinase (BTK) and immune checkpoint $\mathrm{PD}-1$, which regulate tumor growth and metastasis, cannot be ignored. BTK inhibitor (Ibrutinib) and PD-1 blockade in combination with CAR-T therapy may increase $T$ cell survival, and kill PD-L1 positive tumor cells (43). Presently, 20 patients are participating in the herinCAR-PD1 clinical trial for the treatment of EGFR-positive solid tumor since 2016 (NCT02873390). (iii) Indoleamine 2,3-dioxygenase (IDO) is involved in tryptophan metabolism. The tryptophan metabolites result in the inhibition of CAR-T cells proliferation and cytotoxic capability via activating the aryl-hydrocarbon receptor and the expression of the related gene. Besides, it was discovered that the treatment of IDO inhibitor (1-methyl-tryptophan), plus fludarabine and cyclophosphamide, remarkably increased CAR-T anti-tumor efficacy, improved survival rate and decreased IFN- $\gamma$ induced IDO expression (44). Therefore, IDO inhibitors may provide novel therapeutic approaches for CAR-T treatment. (iv) Pembrolizumab is the first-line anti PD-1 monoclonal antibody and it ameliorates the immune suppression mediated by tumor microenvironment. The anti-GD2 CAR-T cell plus pembrolizumab demonstrated a long persistence and limited immune escape (31). (v) It has been observed that tumor vaccines can augment the anti-tumor activity of CAR-T 
cells and contribute to CAR-T cells ablation to prevent their overgrowth. The CMV specific vaccine, which comprises the endogenous CMVpp65 $\mathrm{T}$ cell receptor, significantly improves CAR-T cell persistence and IFN- $\gamma$ secretion without evidence of GVHD. The fraction of CAR-T cells in the total cell population, which was detected by cetuximab, reached nearly $50 \%$ with $120-150$ fold increase (45). (vi) Small molecule inhibitors will also offer novel approaches for tumor treatment because of their efficiency and sensitivity. ABT-737 is a small-molecule inhibitor of Bcl-2, which has been reported to exhibit synergistic effects on CAR-T anti-tumor efficacy. The combination therapy of ABT-737 and anti-CD19 CAR-T cells induces tumor cell apoptosis via caspases 3/7/9 and increases $\mathrm{T}$ cell recognition via cell phenotype changes (46). (III) Preconditioning of CAR-T therapy requires optimization. Currently, leukapheresis, chemotherapy, radiation therapy and autologous hematopoietic stem cell transplantation (HSCT), are the standard strategies to reduce immune rejection and improve survival rate. However, excessive use of IL-2 in preconditioning will consequently aggravate CRS and immune disorder. Therefore, humanization of engraftment, activation of specific CAR-T cells, and selection of appropriate TAAs are the 3 most important challenges that need to be addressed urgently. Besides, the cultivation of CAR-T cells in vitro and promotion of $\mathrm{T}$ cell homing in vivo should also be strengthened to sustain $\mathrm{T}$ cell activity. (IV) Simultaneous and sequential CAR-T cells are being studied for clinical applications. The sequential therapy of CD19 and CD20 bi-specific CAR-T cells was administrated among patients with diffuse large $\mathrm{B}$ cell lymphoma since 2016 (NCT02737085). Besides, EGFR and CD133 bispecific CAR-T cells were infused sequentially in a 52 -yearold female with advanced cholangiocarcinoma. 8.5-month partial response was reported in anti-EGFR therapy and 4.5-month partial response was observed in later anti-CD133 therapy (47). (V) Other ongoing clinical trials are directed at renovating design and introducing on-off molecular switches in initial stage of CAR-T cell construction. Actually, this switch is an apoptosis gene to induce $\mathrm{T}$ cell inactivation and restrain cytokine overproduction, with no interference in the potent anti-tumor activity. Recently, Caspase-9 (iC9) has been introduced as a suicide gene in anti-CD19 CAR design, which not only improves the B cell aplasia, but also demonstrates sustainable remission, great safety and limited toxicity (48). Another apoptosis switch is the WOX1 and MEK interaction. MEK relocates to the lipid raft, and WOX1 transfers to the mitochondria, in which it undergoes phosphorylation and induces apoptosis subsequently (49). This switch may play a crucial role in leukemia, which guides the management of the CAR-T cell overgrowth. In addition, TanCAR could be a positive signal for CAR-T therapy development. By introducing double targets, CAR would be more efficient and sensitive. However, questions such as whether TanCAR can target more tumor antigens or how its design can be improved require further exploration.

\section{Conclusions}

CAR comprises scFvs and co-stimulatory signals to sustain proliferation, enhance cytokine production, and improve scope of anti-tumor response, which has undergone several generations of enhancement. The selection of CAR- $T$ antigens can be summarized according to antigen characteristics. Lesion size was identified with a decrease and prolonged survival time was exhibited among patients who received CAR-T therapy. However, adverse effects cannot be ignored, and should be managed before, during, and after CAR-T therapy. Further investigations regarding CAR-T therapy are required before it can be successfully utilized as a standard strategy for anti-tumor treatment.

\section{Acknowledgments}

We would like to extend gratitude to Professor Yuqin Liu from Chinese Academy of Medical Sciences, who reviewed the content and provided valuable suggestions.

Funding: None.

\section{Footnote}

Conflicts of Interest: Both authors have completed the ICMJE uniform disclosure form (available at http://dx.doi. org/10.21037/tcr.2018.06.11). The authors have no conflicts of interest to declare.

Ethical Statement: The authors are accountable for all aspects of the work in ensuring that questions related to the accuracy or integrity of any part of the work are appropriately investigated and resolved.

Open Access Statement: This is an Open Access article distributed in accordance with the Creative Commons Attribution-NonCommercial-NoDerivs 4.0 International License (CC BY-NC-ND 4.0), which permits the noncommercial replication and distribution of the article with the strict proviso that no changes or edits are made and the original work is properly cited (including links to both the formal publication through the relevant DOI and the license). See: https://creativecommons.org/licenses/by-nc-nd/4.0/. 


\section{References}

1. Orlowski RJ, Porter DL, Frey NV. The promise of chimeric antigen receptor T cells (CARTs) in leukaemia. Br J Haematol 2017;177:13-26.

2. Callahan C, Baniewicz D, Ely B. CAR T-Cell Therapy: Pediatric Patients With Relapsed and Refractory Acute Lymphoblastic Leukemia. Clin J Oncol Nurs 2017;21:228.

3. Fischer J, Paret C, El Malki K, et al. CD19 Isoforms Enabling Resistance to CART-19 Immunotherapy Are Expressed in B-ALL Patients at Initial Diagnosis. J Immunother 2017;40:187-95.

4. Fesnak A, Lin C, Siegel DL, et al. CAR-T Cell Therapies From the Transfusion Medicine Perspective. Transfus Med Rev 2016;30:139-45.

5. Ramanayake S, Bilmon I, Bishop D, et al. Low-cost generation of Good Manufacturing Practice-grade CD19specific chimeric antigen receptor-expressing $\mathrm{T}$ cells using piggyBac gene transfer and patient-derived materials. Cytotherapy 2015;17:1251-67.

6. Sun M, Shi H, Liu C, et al. Construction and evaluation of a novel humanized HER2-specific chimeric receptor. Breast Cancer Res 2014;16:R61.

7. Wang J, Jia R, Yao Y, et al. Differential expression of Mart-1 in human uveal melanoma cells. Mol Med Rep 2011;4:799-803

8. Cretella D, Saccani F, Quaini F, et al. Trastuzumab emtansine is active on HER-2 overexpressing NSCLC cell lines and overcomes gefitinib resistance. Mol Cancer 2014;13:143.

9. Ahmed N, Brawley VS, Hegde M, et al. Human Epidermal Growth Factor Receptor 2 (HER2) -Specific Chimeric Antigen Receptor-Modified T Cells for the Immunotherapy of HER2-Positive Sarcoma. J Clin Oncol 2015;33:1688-96.

10. Zhou F, Krishnamurthy J, Wei Y, et al. Chimeric antigen receptor T cells targeting HERV-K inhibit breast cancer and its metastasis through downregulation of Ras. Oncoimmunology 2015;4:e1047582.

11. Anurathapan U, Chan RC, Hindi HF, et al. Kinetics of tumor destruction by chimeric antigen receptor-modified T cells. Mol Ther 2014;22:623-33.

12. Gargett T, Fraser CK, Dotti G, et al. BRAF and MEK inhibition variably affect GD2-specific chimeric antigen receptor (CAR) T-cell function in vitro. J Immunother 2015;38:12-23.

13. Morgan RA, Johnson LA, Davis JL, et al. Recognition of glioma stem cells by genetically modified $\mathrm{T}$ cells targeting EGFRvIII and development of adoptive cell therapy for glioma. Hum Gene Ther 2012;23:1043-53.

14. Krebs K, Böttinger N, Huang LR, et al. T cells expressing a chimeric antigen receptor that binds hepatitis $\mathrm{B}$ virus envelope proteins control virus replication in mice. Gastroenterology 2013;145:456-65.

15. Nakazawa Y, Huye LE, Salsman VS, et al. PiggyBacmediated cancer immunotherapy using EBV-specific cytotoxic T-cells expressing HER2-specific chimeric antigen receptor. Mol Ther 2011;19:2133-43.

16. Wang W, Ma Y, Li J, et al. Specificity redirection by CAR with human VEGFR-1 affinity endows T lymphocytes with tumor-killing ability and anti-angiogenic potency. Gene Ther 2013;20:970-8.

17. Schuberth PC, Jakka G, Jensen SM, et al. Effector memory and central memory NY-ESO-1-specific redirected $\mathrm{T}$ cells for treatment of multiple myeloma. Gene Ther 2013;20:386-95.

18. Cheadle EJ, Rothwell DG, Bridgeman JS, et al. Ligation of the CD2 co-stimulatory receptor enhances IL-2 production from first-generation chimeric antigen receptor T cells. Gene Ther 2012;19:1114-20.

19. Suryadevara CM, Desai R, Farber SH, et al. 369 Chimeric Antigen Receptors Deficient in Lck Signaling Require 4-1BB Costimulation to Expand in Vivo, Resist Regulatory T-Cell Suppression, and Treat Solid Tumors in ImmuneIntact Hosts. Neurosurgery 2016;63 Suppl 1:209-10.

20. Tang XY, Sun Y, Zhang A, et al. Third-generation CD28/4-1BB chimeric antigen receptor $T$ cells for chemotherapy relapsed or refractory acute lymphoblastic leukaemia:a non-randomised, open-label phase I trial protocol. BMJ Open 2016;6:e13904.

21. Chmielewski M, Abken H. TRUCKs:the fourth generation of CARs. Expert Opin Biol Ther 2015;15:1145-54.

22. Pegram HJ, Smith EL, Rafiq S, et al. CAR therapy for hematological cancers:can success seen in the treatment of B-cell acute lymphoblastic leukemia be applied to other hematological malignancies? Immunotherapy 2015;7:545-61.

23. Hegde $M$, Mukherjee $M$, Grada Z, et al. Tandem CAR T cells targeting HER2 and IL13Ralpha2 mitigate tumor antigen escape. J Clin Invest 2016;126:3036-52.

24. Schneider D, Xiong Y, Wu D, et al. A tandem CD19/ CD20 CAR lentiviral vector drives on-target and off-target antigen modulation in leukemia cell lines. J Immunother Cancer 2017;5:42.

25. Wang X, Popplewell LL, Wagner JR, et al. Phase 1 studies of central memory-derived CD19 CAR T-cell therapy following autologous HSCT in patients with B-cell NHL. Blood 2016;127:2980-90. 
26. Rossig C, Pule M, Altvater B, et al. Vaccination to improve the persistence of CD19CAR gene-modified T cells in relapsed pediatric acute lymphoblastic leukemia. Leukemia 2017;31:1087-95.

27. Minagawa K, Jamil MO, Al-Obaidi M, et al. In Vitro PreClinical Validation of Suicide Gene Modified Anti-CD33 Redirected Chimeric Antigen Receptor T-Cells for Acute Myeloid Leukemia. PLoS One 2016;11:e0166891.

28. Ramos CA, Ballard B, Zhang H, et al. Clinical and immunological responses after CD30-specific chimeric antigen receptor-redirected lymphocytes. J Clin Invest 2017;127:3462-71.

29. Junghans RP, Ma Q, Rathore R, et al. Phase I Trial of Anti-PSMA Designer CAR-T Cells in Prostate Cancer:Possible Role for Interacting Interleukin 2-T Cell Pharmacodynamics as a Determinant of Clinical Response. Prostate 2016;76:1257-70.

30. Thistlethwaite FC, Gilham DE, Guest RD, et al. The clinical efficacy of first-generation carcinoembryonic antigen (CEACAM5)-specific CAR T cells is limited by poor persistence and transient pre-conditioning-dependent respiratory toxicity. Cancer Immunol Immunother 2017;66:1425-36.

31. Gargett T, Yu W, Dotti G, et al. GD2-specific CAR T Cells Undergo Potent Activation and Deletion Following Antigen Encounter but can be Protected From Activationinduced Cell Death by PD-1 Blockade. Mol Ther 2016;24:1135-49.

32. Morgan RA, Yang JC, Kitano M, et al. Case report of a serious adverse event following the administration of T cells transduced with a chimeric antigen receptor recognizing ERBB2. Mol Ther 2010;18:843-51.

33. Shank BR, Do B, Sevin A, et al. Chimeric Antigen Receptor T Cells in Hematologic Malignancies. Pharmacotherapy 2017;37:334-45.

34. Mackall CL, Miklos DB. CNS Endothelial Cell Activation Emerges as a Driver of CAR T Cell-Associated Neurotoxicity. Cancer Discov 2017;7:1371-3.

35. Maus MV, Haas AR, Beatty GL, et al. T cells expressing chimeric antigen receptors can cause anaphylaxis in humans. Cancer Immunol Res 2013;1:26-31.

36. Wilson FP, Berns JS. Tumor lysis syndrome:new challenges and recent advances. Adv Chronic Kidney Dis 2014;21:18-26.

37. Gust J, Hay KA, Hanafi LA, et al. Endothelial Activation and Blood-Brain Barrier Disruption in Neurotoxicity after Adoptive Immunotherapy with CD19 CAR-T Cells. Cancer Discov 2017;7:1404-19.

38. Brentjens R, Yeh R, Bernal Y, et al. Treatment of chronic lymphocytic leukemia with genetically targeted autologous $T$ cells:case report of an unforeseen adverse event in a phase I clinical trial. Mol Ther 2010;18:666-8.

39. Tasian SK, Kenderian SS, Shen F, et al. Optimized depletion of chimeric antigen receptor $\mathrm{T}$ cells in murine xenograft models of human acute myeloid leukemia. Blood 2017;129:2395-407.

40. Lo A, Wang LS, Scholler J, et al. Tumor-Promoting Desmoplasia Is Disrupted by Depleting FAP-Expressing Stromal Cells. Cancer Res 2015;75:2800-10.

41. Mata M, Gerken C, Nguyen P, et al. Inducible Activation of MyD88 and CD40 in CAR T Cells Results in Controllable and Potent Antitumor Activity in Preclinical Solid Tumor Models. Cancer Discov 2017;7:1306-19.

42. Caruana I, Savoldo B, Hoyos V, et al. Heparanase promotes tumor infiltration and antitumor activity of CAR-redirected T lymphocytes. Nat Med 2015;21:524-9.

43. Molica S. Ibrutinib continues to influence the therapeutic landscape of chronic lymphocytic leukemia:new data presented at ASCO 2017. BMC Med 2017;15:156.

44. Ninomiya S, Narala N, Huye L, et al. Tumor indoleamine 2,3-dioxygenase (IDO) inhibits CD19-CAR T cells and is downregulated by lymphodepleting drugs. Blood 2015;125:3905-16.

45. Wang X, Wong CW, Urak R, et al. CMVpp65 Vaccine Enhances the Antitumor Efficacy of Adoptively Transferred CD19-Redirected CMV-Specific T Cells. Clin Cancer Res 2015;21:2993-3002.

46. Karlsson H, Lindqvist AC, Fransson M, et al. Combining CAR T cells and the Bcl-2 family apoptosis inhibitor ABT737 for treating B-cell malignancy. Cancer Gene Ther 2013;20:386-93.

47. Feng KC, Guo YL, Liu Y, et al. Cocktail treatment with EGFR-specific and CD133-specific chimeric antigen receptor-modified $\mathrm{T}$ cells in a patient with advanced cholangiocarcinoma. J Hematol Oncol 2017;10:4.

48. Diaconu I, Ballard B, Zhang M, et al. Inducible Caspase-9 Selectively Modulates the Toxicities of CD19-Specific Chimeric Antigen Receptor-Modified T Cells. Mol Ther 2017;25:580-592.

49. Lin HP, Chang JY, Lin SR, et al. Identification of an In Vivo MEK/WOX1 Complex as a Master Switch for Apoptosis in T Cell Leukemia. Genes Cancer 2011;2:550-62.

Cite this article as: $\mathrm{Ni} \mathrm{J,} \mathrm{Ni} \mathrm{A.} \mathrm{Chimeric} \mathrm{antigen} \mathrm{receptor-T}$ cell, a novel player in anti-tumor treatment. Transl Cancer Res 2018;7(4):1143-1150. doi: 10.21037/tcr.2018.06.11 